\title{
ANALYSIS OF THE AVAILABILITY OF FOREST BIOMASS AS AN INPUT FOR THE GENERATION OF SUSTAINABLE ENERGY IN A COMPANY IN THE TEXTILE SECTOR OF THE ABURRA VALLEY
}

\section{MARTHA OSPINA HERNANDEZ ${ }^{1}$, LEONARDO MARTINEZ RIOS ${ }^{1}$, SIR-ALEXCI SUAREZ ${ }^{2}$ \&}

\section{ALBERT MIYER SUAREZ ${ }^{3}$}

${ }^{1}$ School of Basic Sciences, Technology and Engineering, National Open and Distance University UNAD, Colombia

${ }^{2}$ Engineering Faculty, University Francisco of Paula Santander Ocaña, Colombia.

${ }^{3}$ Engineering Faculty. University of Pamplona, Colombia.

\begin{abstract}
The new world energy policies seek to reduce dependence on hydrocarbons and their derivatives as the main energy resource, given their condition as a limited resource and the environmental impacts derived from their application. Colombia, for its part, depends to a great extent on fossil resources, which have a reserve / production ratio of 5.9 years for oil, 14 years for natural gas and coal, although it is valid for at least 79 years. If the rate of exploitation continues it is not the cleanest way to generate electricity. That said, it is intended that this study provides information for innovative agents and entrepreneurs in the field of renewable energies, especially in wood energy and its application mainly in the department of Antioquia.
\end{abstract}

KEYWORDS: Biomass, Forest Biomass, Wood Energy, Renewable Energy, Logistics, Power Generation

Received: Nov 05, 2020; Accepted: Nov 25, 2020; Published: Dec 04, 2020; Paper Id.: IJMPERDDEC20204

\section{INTRODUCTION}

The company under study has been self-generating a significant percentage (88\%) of its demand for electricity through a coal-based thermoelectric plant that generates $14 \mathrm{MW} / \mathrm{h}$, due to the need to self-supply $100 \%$ of its energy need, To remain competitive in a globalized market, the company is interested in increasing its installed capacity through a new generating plant, for this new project what is sought is that this need be satisfied in a sustainable way, following the line of the high environmental responsibility that the company has strictly ingrained. For this purpose, the company is investigating the possibilities of generating electrical energy using biomass as raw material for combustion.

This work will focus on studying the capacity and costs of supplying biomass or organic matter in the region, specifically in a radius of 50 to 100 kilometers from the company, thus the work carried out will allow the company to know which are the most viable materials depending on its available quantity, acquisition costs, freight and other efficiency factors such as calorific value or humidity percentage, as well as establishing possible strategic allies in production and supply. According to the literature, these perimeters should ideally be 50 kilometers around the company, this due to logistics costs, they also recommend that a second line should be made where it covers up to 100 kilometers, a perimeter within which suppliers could be found that could be reached to supply agreements with competitive logistics costs. Identification of the 50-kilometer perimeter of the company under study. In this perimeter is mainly the valley of the bora, where the largest concentration of sawmills and the main offices of the largest 
reforestation plants in Antioquia are located. On the subject of possible competition for forest resources at an industrial level, it can be said that there are many large companies that could venture into this renewable energy alternative in the future, but within this perimeter they are only found in addition to the company under study two self-generating energy companies, which are also in the textile area, emphasizing that these two generate their energy with steam from coal. Another company within this perimeter that ventures into power generation is one of the food sector located in Rionegro, but it is different from those previously mentioned, they have a solar energy distribution network (photovoltaic generation) with the capacity to generate 2, $2 \mathrm{MW}$ (megawatts) that represent between 15 and 20\% of its energy demand, Identification of the perimeter of 100 kilometers of the company under investigation. It should be noted that the importance of the demarcation and identification of this perimeter goes beyond the industrial competition for the forest resource and is framed more by the forest biomass resources, although important areas of Antioquia where there is a large presence of hectares with Trees planted for reforestation, such as the Urabá Antioqueño and the Bajo Cauca are outside these limits, if there are great opportunities with the existing crops within this perimeter such as the great part of the Northeast, Southwest, North and West of Antioquia, where large reforestation companies are present. Investigation of companies within established geographical limits. Using the Google, search engine, Maps and Earth tools, a list of companies is made with suggestive profiles of possible suppliers of forest biomass, such as sawmills, manufacturers of wood-based products, distributors and reforesters, in addition to the identification of the company, their telephone contact numbers, the distance from the company, their web pages and their emails (in the case of having them) are recorded. Being consistent with the availability of biomass in the region (Perez \& Osorio, 2014), the usable energy in a pilot program for the generation of electricity in the country, would be in the order of $5000 \mathrm{KWe}$, that is, a range of low and medium powers, power that will require a forest nucleus of 5000 ha (hectares) that allocates its production exclusively for the purpose of generating electricity, otherwise it must be incurred in the collection of forest biomass residues from different sources (sawmills, reforestation plants, etc.), a situation in which the issue of logistics costs must be analyzed with special care, since these are above $40 \%$ of costs.

Supply chain - Transportation management: The transportation of biomass according to the geographical location of the company and the possible supply centers must be by land, it is an aspect of greater relevance since it is the link that articulates the use forestry with the factory, is of vital importance for the intentions of energy use for the purpose of generating electricity, as it represents between 50 and $70 \%$ of the increase in the total value of wood in the factory (Gutiérrez et al, 2013).

As the recommendation is to transport the largest amount of biomass to reduce transport costs and it is understood that in the form of logs the transport is optimized and made more efficient, the formulas for cubing and measuring the wood in this presentation are shared (Gutiérrez et al, 2013).

\section{METHODOLOGY}

Because the objective of this research is to analyze the availability of forest biomass for energy purposes in the municipality of Girardota Antioquia, a non-experimental design applied in a transversal way was used. Starting from the fact that the research topic has broad theoretical support in general terms and some studies in the region (not Girardota itself), a descriptive-explanatory research was carried out, to properly know what forest biomass is, it's more general characteristics and its applications supported by instruments that will be mentioned later to determine the possibilities of obtaining this resource.

According to non-experimental research "is that which is done without deliberately manipulating the variables. 
What is done in this type of research is to observe phenomena as they occur in a natural context, and then analyze them" (Fernandez et al, 2020, p.270). The same authors state that cross-sectional research designs "collect data in a single moment, in a single time. Its purpose is to describe variables and analyze their incidence and interrelation at a given moment" (Fernandez et al, 2020, p.289).

This research was designed under the methodological approach of a mixed approach (quantitative-qualitative), because it is an investigation where some aspects such as chemical characteristics, costs and others must be argued with figures. Qualitative in that it comprises a study of other studies carried out and from there create definitions or show the results of the research carried out, it also focuses on the collection of verbal information obtained first-hand that is subjectively analyzed, "It is often based in data collection methods without numerical measurement, such as descriptions and observations. Its purpose is to "rebuild" reality, as observed by the actors of a previously defined social system" (Fernandez et al, 2020, p.5). The quantitative approach assumes information that can be measured and "relies on numerical measurement, counting, and often the use of statistics to accurately establish patterns of behavior in a population." (Fernandez et al, 2020, p.5).

The research participants were representatives of specialized companies in the sector, engineers and experts in the field who contacted each other individually to obtain relevant information through interviews and surveys. In addition, understanding that the population is defined as "a set of all the elements that we are studying, about which we try to draw conclusions" (Levin et al, 1996, p.20), it is stated that the study population is made up of 20 cultivating companies (reforestation plants) and the wood transformation sector.

"The statistical sample is a set of computerized measurements of sample data. Population parameters are characteristic measures of a specific population and statistical samples are used to make inferences (assumptions) about population parameters. " (Zikmund \& Babin, 2009). As mentioned in the previous section, the study population is made up of 20 companies, which in advance supposes a small population, however, it should be taken into account that for the quantities of biomass required, only medium and large ones are considered mainly. Companies, since the supply capacity of the input will be proportional to the size of their operations. There are small growers and sawmills that produce the resource, but they depend on companies (included in the statistical study) that are in charge of collecting biomass from these small producers in order to market it later. With this clear, we proceed to calculate the sample size for a finite and known population with equation (1). A finite population is understood to be one that can be physically enumerated.

$$
n=\frac{Z^{2} * \mathrm{p} * \mathrm{q} * \mathrm{~N}}{e^{2}(\mathrm{~N}-1)+Z^{2} * \mathrm{p} * \mathrm{q}}
$$

Where:

$$
\begin{aligned}
& \mathrm{n}=\text { sample size } \\
& \mathrm{p}=\text { probability in favor }-0.5 \text { (It is recommended to put } 50 \% \text { for not having a history) } \\
& \mathrm{q}=\text { probability against }-0.5 \text { (It is recommended to put } 50 \% \text { for not having a history) } \\
& \mathrm{Z}=\text { confidence level }-1.62 \text { (For a confidence level of } 90 \% \text { ) } \\
& \mathrm{e}=\text { allowed error }-0.2 \\
& \mathrm{~N}=\text { population size }-20
\end{aligned}
$$


Thus, the value of the sample is 10 companies, it should be noted that because the management of forest residues is not yet an item that is monitored in the accounts of all companies, and given the incipient implementation technologies for the use of biomass and agents interested in the resource; the value used for the allowed error (e) corresponds to $20 \%$, thus achieving aligning the strategy with the data obtained. The 10 companies are selected with an intentional non-probability sampling strategy.

\section{Instruments}

To determine the universe to be investigated in the research in terms of supply of the resource, an investigation is carried out on the internet on the primary sources that could become providers of information on the supply capacity of a possible wood-energy project, for this a list of the wood cultivating and processing companies in the region and a questionnaire with 4 multiple-choice questions is applied, each of them exposed in the next section.

\section{Data Analysis Strategy}

The technique used to process the data obtained is descriptive statistics, with respect to this it infers that, "the records or observations made provide a series of data that must necessarily be ordered and presented in an intelligible way. Descriptive statistics develops a set of techniques whose purpose is to present and reduce the different observed data"

To interpret the data obtained through the surveys carried out, as well as the other data collected during the investigation, Microsoft Office Excel software was used.

\section{RESULTS AND DISCUSSIONS}

Taking into account that pyrolysis and liquefaction are technologies that are under development, it is concluded that the two technically feasible technologies for the energy conversion of forest biomass in the country are gasification and combustion. Gasification is a technology with low installation costs. Furthermore, the reactors can be used with forest biomass or biodigesters could be installed to use biomass from organic waste, without presenting major changes in the rest of the process; however, the disadvantage is the high maintenance costs due to the resulting gases that must be filtered to avoid fouling or fouling in alternative internal combustion engines (MCIA). For its part, although combustion involves higher installation costs, it has the advantage that facilities used for the combustion of coal or other types of biomass can be used in a mixed way, in the case of being implemented in a system designed for the combustion of coal, it would present very important reductions in the emissions of polluting gases. On the other hand (Cabrera et al, 2012), the calorific power of biomass is lower than that of coal, sustaining the same energy generation conceived by the original design based on biomass, implies greater amounts of fuel supply. $\mathrm{SO}_{x}, \mathrm{NO}_{x}, \mathrm{CO}$ y $\mathrm{CO}_{2}$

Continuing with the determinations of the calorific power, an aspect of vital relevance for the viability of energy generation projects from forest biomass, due to its relationship with the amount of energy that each particle of biomass supplies to the process and what it implies This aspect in the quantities to be required and consequently transported, it is observed that there is not a great difference in the calorific powers of the species available in the region, which means that the species is not a determining factor for the time to choose the best input, other than the humidity percentage, which does have an effect on the calorific value of the wood (see figure 1).

In contrast, from the surveys carried out to the representatives of companies with a profile of possible suppliers of forest resources in the area, the following results are obtained: 
To the question, what is the amount of wood waste per day generated in your processes?

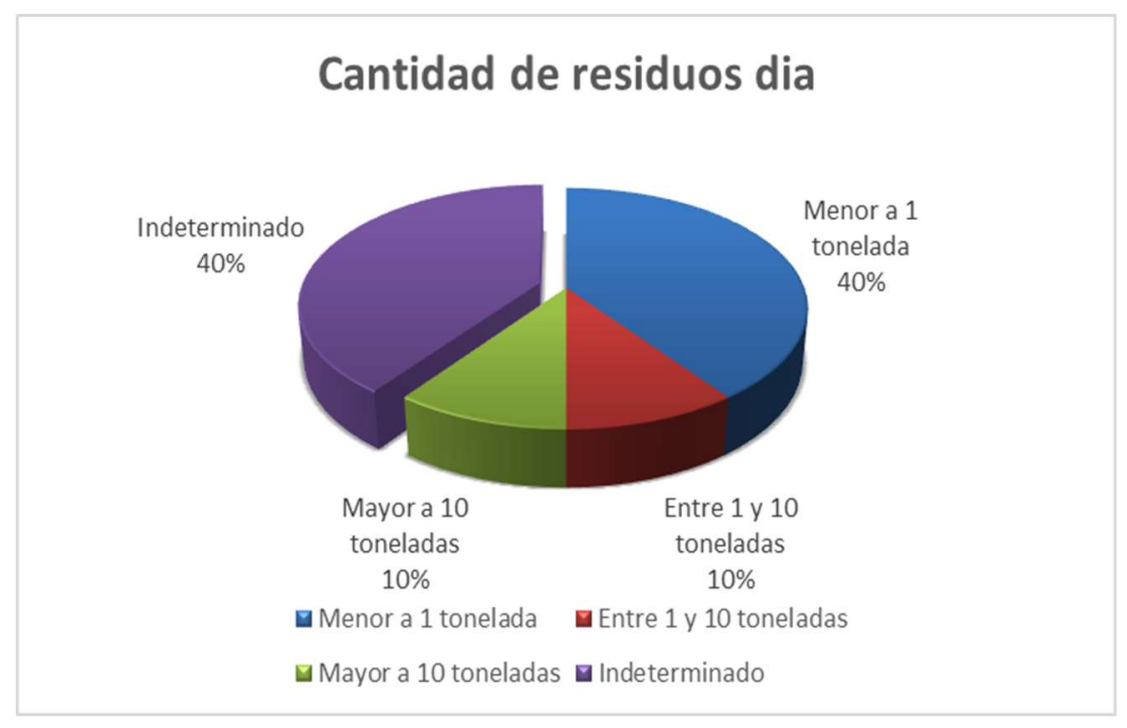

Figure 1: Circular diagram to question about the amount of wood waste per day

It is striking that the majority of responses correspond to the fact that the residues are the equivalent of one ton or less and the indeterminacy of these residues; For the responses of waste equal to or less than one ton, it can be deduced that it is because a high percentage of the companies with which it was possible to establish communication are sawmills and since their intention is to convert the wood into other by-products, Added to the improvements in technologies and methods to minimize waste, these are usually very low for the intentions of generating electricity from this resource. On the other hand, the high percentage of companies that do not know the amount of their waste stands out (see Figure 2).

To the question, where are your silvicultural crops located?

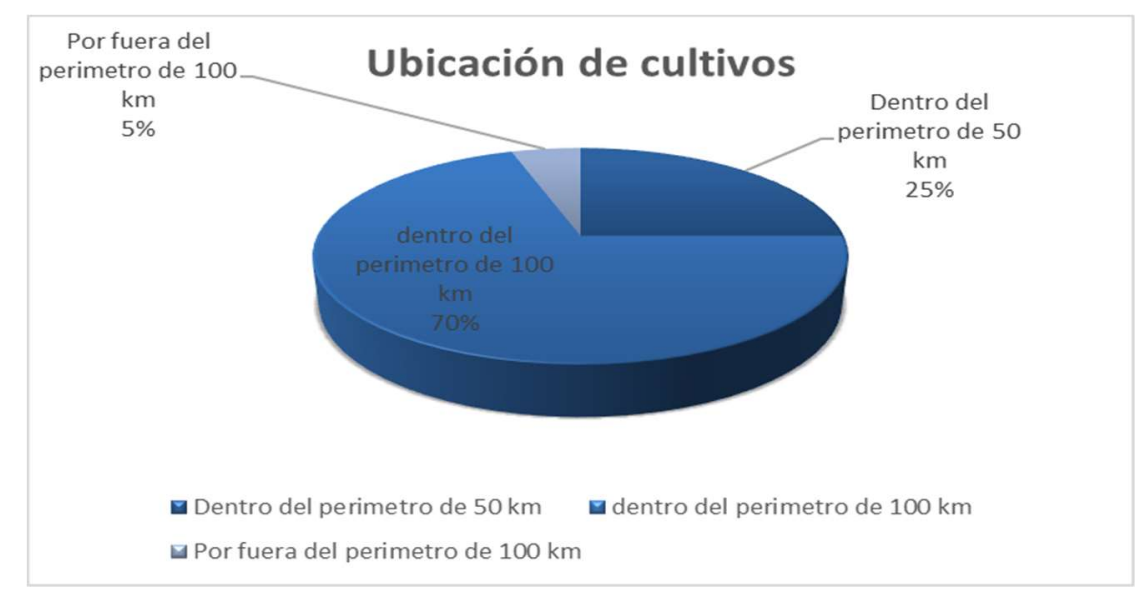

Figure 2: Circular diagram on the location of silvicultural crops

This question focuses on determining the origin of the crops that provide the companies with their raw material and is intentionally directed to knowing the distance of these crops from the origin to the processing centers, starting from the elementary premise subtracted from the related bibliographies. On the recommended distances in terms of logistics efficiency. It is highlighted that the highest density of responses coincides in that the supply is obtained with crops located within the perimeter of 100 kilometers of the processing center, this is because the largest silvicultural crops are located in 
the Northeast, Southwest, North and West from Antioquia, where the most important reforestation companies are located (see Figure 3).

To the question, what are the types of wood that you use in your processes?

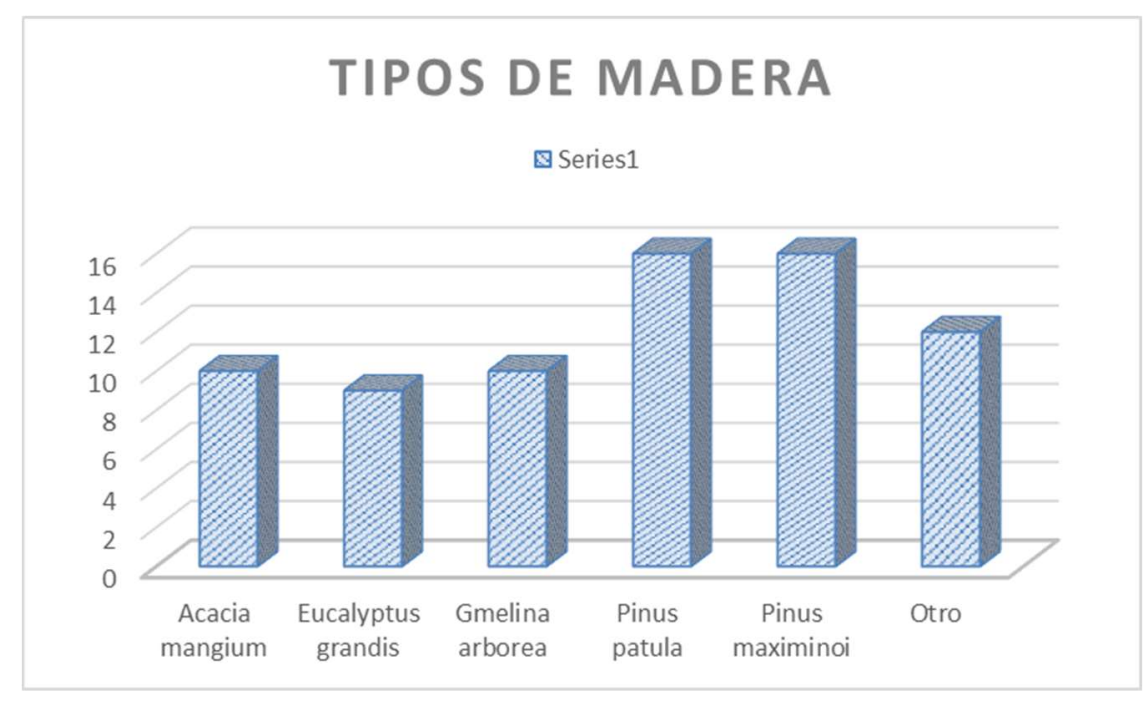

Figure 3: Column diagram on the types of wood used

As mentioned, these five species are the predominant ones in the region and have very similar characteristics in terms of energy potential. There is a slight inclination towards pine species, due to the fact that this species has a high affinity with the soils of the region; the item "other" refers to other species used for jobs or special products (teak, cedar, guayacán, etc.) But that are not necessarily native or related to the region or that their analysis is not deepened due to their lower energy density (see Figure 4).

To the question, what is the cost of the final disposal of your forest and process residues?

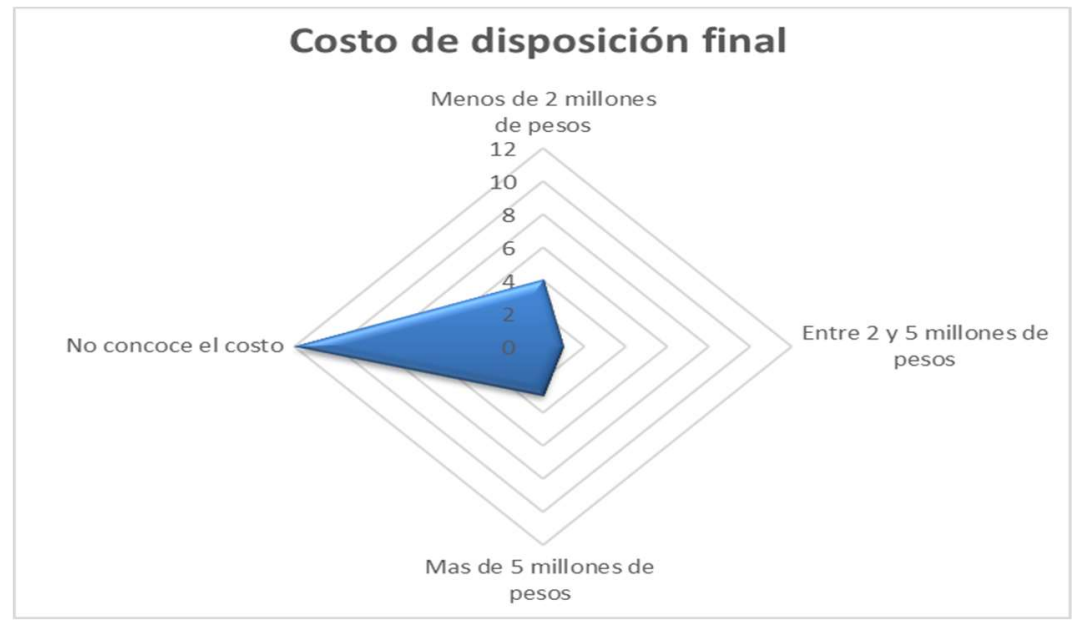

Figure 4: Radar chart on the final disposal costs of wood waste

It can be seen that companies are largely unaware of the cost of the final disposal of their waste, this condition occurs because the companies sell or dispose of their waste so that a company dedicated exclusively to collecting this waste can collect it at the collection sites temporary, assuming transportation costs, which leads to ignorance of the cost of disposal. 
On the other hand, companies that generate waste from pruning or felling trees, if they incur disposal costs due to the fact that for them this waste does not have an application, this condition represents an opportunity because these waste have important energy contents that can be taken advantage of.

\section{CONCLUSIONS}

Forest biomass is a renewable energy resource with great potential at this time where it is essential to migrate to new (nonfossil) energies that are efficient and friendly to the environment. Colombia and particularly Antioquia have great potential in terms of wood energy, unfortunately there are still no great advances in terms of implementation based on their silvicultural potential, since with current crops it is possible to undertake medium power energy generation projects (up to $5 \mathrm{MWe}$ ), the implementer of wood-energy projects has widely tested and verified existing technologies at hand to exploit forest resources, among them gasification and combustion stand out,

From the data collection management of the possible suppliers of forest biomass, it can be concluded that there is a large company that is the one that uses the forest biomass resource the most in the investigated perimeter, this finding can be explained by its high demand for the generation of thermal energy for processes (180 ton (/ day), which indicates that an entrepreneur of projects related to wood energy in the investigated region should consider making commercial alliances with this giant of forest resources, to guarantee the supply of biomass for your processes.

In terms of logistics and transportation, it can be concluded that, although the main alternative is land transportation, the alternative of moving the generation plant to the vicinity of the reforestation areas and from there transporting the energy through electrical networks should also be analyzed. . However, if you want to use the traditional method, it is vitally important to optimize the weight / volume transported ratio, for this it is recommended as a first measure, transporting the wood by truck, given its greater load capacity (32 tons) and making use mainly of round wood because due to its higher density it can be transported much more; This option requires that a chipping plant be installed or this service be outsourced,

Finally, it is important to note that according to agreement 092 of November 30, 2007, in which the basic land use plan for the municipality of Girardota is reviewed and adjusted, it is stated that tol at least $30 \%$ of the lands of the rural plots in the future must be used for the planting of native trees adapted to their specific problems and that of the region. Additionally, to date, this plan has materialized in the planting of more than 67,000 trees in the municipality since 2016, in addition to the 35 hectares of green area that have been acquired in the area. If the appropriate forestry techniques are used, this can mean the assurance of a significant part of the demand for the energy input and reduce transport logistics costs, translating into supply guarantees that can give high viability to projects of this type.

\section{REFERENCES}

1. Pérez, and LF Osorio, "Forest biomass as an energy alternative, silvicultural, technical and financial analysis of projects (Editorial)." Medellin, 2014.

2. Gutiérrez, R. Moreno, and N. Villota, "Wood cubing guide. [Digital book] ", 2013 [Online].Available: http://www.rivasdaniel.com/pdf/guia_de_cubicacion_madera.pdf

3. Fernández, JMC Sánchez, A. Córdoba, and AC Largo, "Descriptive Statistics", Esic Editorial., 2002.

4. Levin, D. Rubin, and A. Samaniego, "Statistics for administrators", 1996 [Online]. Available: http://dspace.ucbscz.edu.bo/dspace/bitstream/123456789/4294/3/584.pdf 
5. Zikmund, and BJ Babin, "Market Research", Cencage learning editors. SA., 9 ed., 2009.

6. Cabrera, S. Madriñan, and D. Muñoz, "Characterization of gas obtained with the algarrobo and sugarcane bagasse", Biotechnology in the Agricultural and Agroindustrial Sector, vol. 10, 2012. 